\title{
New Ultra-High Resolution SEM for Imaging by Low Energy Electrons
}

\author{
Jaroslav Jiruše, Miloslav Havelka, Jan Polster and Filip Lopour
}

TESCAN a.s., Brno, Czech Republic

Charging of non-conductive materials, observation of very thin layers, damaging of sensitive samples these are the problems that many scientists are faced with using scanning electron microscopy (SEM) in biology, bioengineering, nanosensor or semiconductor research. Conventional microscopes use high energy electrons $(>10 \mathrm{keV})$ in order to get high resolution of about $1 \mathrm{~nm}$. However, high energy electrons can cause charging of non-conductive materials, penetrate deep through the upper layers of the sample, can break down bonds between molecules of biological tissues and polymers and damage the sample. To observe the above-mentioned materials it is advisable to use electrons with low energies. However, in SEMs fitted with a conventional objective lens, the decrease of electron energy increases aberrations of the optical system and makes determination of very fine details problematic. An instrument with high-resolution imaging at low energies is preferred.

TESCAN has developed a new analytical microscope, MAIA, with a high resolution of $1 \mathrm{~nm}$ at $15 \mathrm{kV}$ and $1.6 \mathrm{~nm}$ at $1 \mathrm{kV}$ with secondary electrons, and $0.8 \mathrm{~nm}$ at $30 \mathrm{kV}$ in STEM mode. This instrument is based on a three-lens column equipped with a Schottky field-emission gun. The objective lens is based on a single-pole immersion type [1] that decreases optical aberrations dramatically compared to a conventional lens. An additional intermediate lens can operate simultaneously with, or instead of, the objective lens. Thus multiple display modes are possible (e.g. for best resolution, largest field of view or greatest depth of focus). This also enables a field-free mode.

TESCAN's beam deceleration technology can also be used in the new MAIA, allowing automatic control of the electron landing energy down to $50 \mathrm{eV}$ (or in manual regime down to $0 \mathrm{eV}$ ) by applying negative voltage on the sample. This regime also helps in charge-free imaging. The new module provides for sample discharge-and-touch protection, and switches between deceleration and normal regimes automatically without the necessity of any manual operation. Very accurate computation of working distance, magnification and all optical parameters together with continuous control of the beam spot size and beam current is ensured by In-Flight Beam Tracing ${ }^{\mathrm{TM}}$ technology.

The detection system includes innovative In-Beam SE, In-Beam BE, and SE in beam deceleration mode, all of which are in-lens (in column) detectors that provide variety of signals in addition to standard SE, $\mathrm{BE}$ or TE detectors in the chamber.

The instrument is able to achieve probe currents up to $200 \mathrm{nA}$, suitable for analytical methods such as EDX, WDX, EBSD, CL etc. All of these detectors are optimized to work at a short analytical working distance of $5 \mathrm{~mm}$.

E-beam lithography benefits from TESCAN's specialized DrawBeam software which provides a familiar CAD-like GUI for drawing of all shapes, together with ultra-fast scanning (pixel dwell times down to $20 \mathrm{~ns}$ ). Fast and efficient microscope control with many automatic functions (focusing, stigmation, brightness, centering, spot optimization, auto-diagnostics and many more) and software extensions (image processing, 3D scanning, correlating position with photograph or light optical image 
etc.) enables a real user-friendly operation [2].

[1] Z Shao, PSD Lin, Rev. Sci. Instrum. 60(11) (1989) p. 3434.

[2] The authors acknowledge funding from Technological Agency of Czech Republic under grant FRTI2/736, project Moremit.
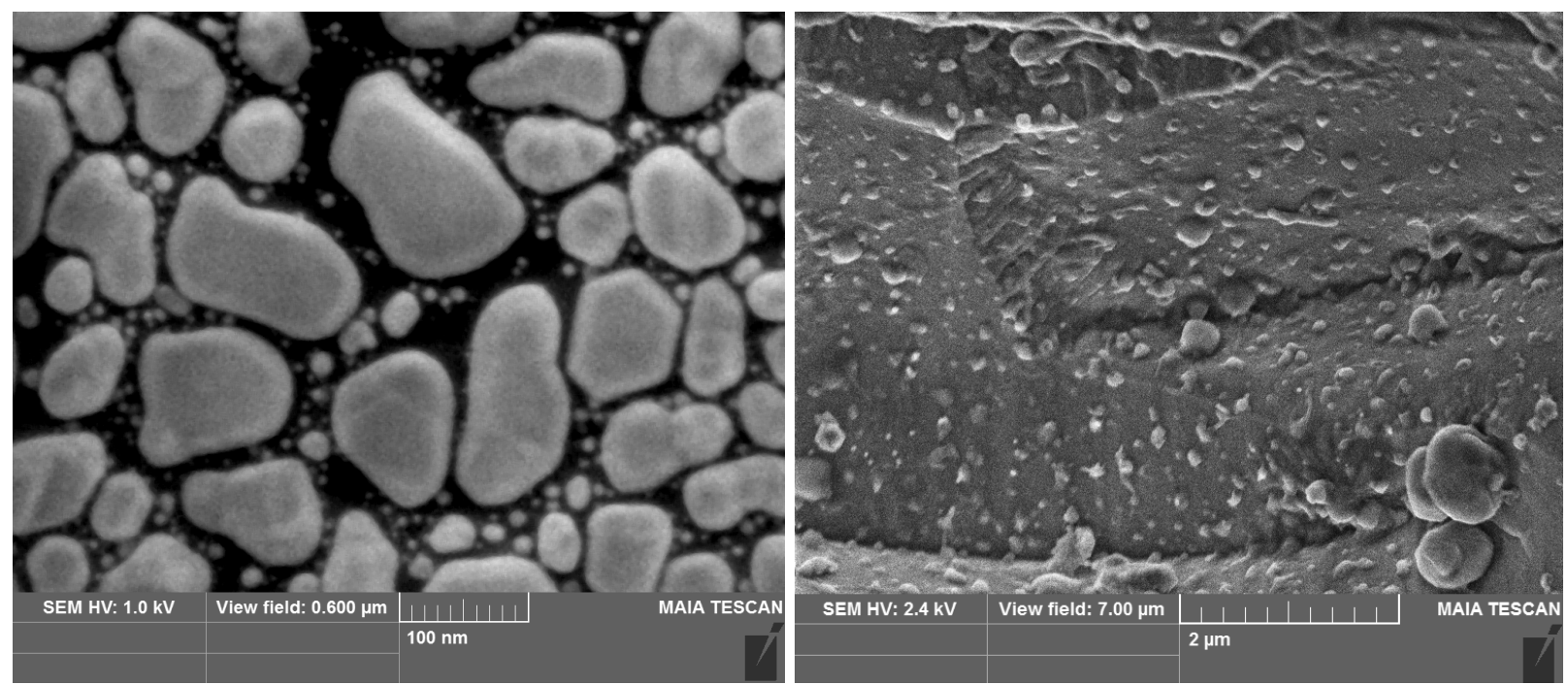

Figure 1. A resolution test sample of gold on carbon at $1 \mathrm{kV}$ (left) and non-conductive particles on glass (right). 